\title{
Novel hybrid process for the conversion of microcrystalline cellulose to value-added chemicals: part 2: effect of constant voltage on product selectivity
}

\author{
Okan Akin • Asli Yuksel
}

Received: 3 May 2017/ Accepted: 14 August 2017/Published online: 22 August 2017

(C) Springer Science+Business Media B.V. 2017

\begin{abstract}
In this study, electrochemical degradation of microcrystalline cellulose (MCC) under hot-compressed water was investigated via application of constant voltage on reaction medium. Constant voltage ranges from 2.5 to $8.0 \mathrm{~V}$ was applied between anode (Titanium) and cathode (reactor wall). As an electrolyte and proton source 5-25 $\mathrm{mM}$ of $\mathrm{H}_{2} \mathrm{SO}_{4}$ was used. Reactions were carried out in a specially designed batch reactor $(450 \mathrm{~mL})$ made of $\mathrm{T} 316$ for $240 \mathrm{~min}$ at temperature of $200{ }^{\circ} \mathrm{C} . \mathrm{MCC}$ decomposition products such as glucose, fructose, furfural, 5-HMF and levulinic acid were detected and quantified by High Performance Liquid Chromatography (HPLC). In the absence of electrolyte, applied voltage $(2.5$ and $4.0 \mathrm{~V})$ decreased the total organic carbon (TOC) yield, in contrast at $8.0 \mathrm{~V}$, TOC yield increased to $13 \%$. Application of $8.0 \mathrm{~V}$ in hydrothermal conditions alter MCC decomposition pathway selectively to furfural $(15 \%)$. Addition of electrolyte $(5 \mathrm{mM}$, $\mathrm{H}_{2} \mathrm{SO}_{4}$ ) and application of $2.5 \mathrm{~V}$ potential increased TOC (54\%) and changed the decomposition pathway in favor of 5-HMF (30\%) and levulinic acid (21\%). The structural changes in solid residues of electrochemically reacted MCC was analyzed by Fourier Transform Infrared Spectroscopy (FTIR) and found that MCC particles functionalized by carboxylic acid
\end{abstract}

O. Akin · A. Yuksel ( $\square)$

Chemical Engineering Department, İzmir Institute of Technology, 35430 Urla, Izmir, Turkey

e-mail: asliyuksel@iyte.edu.tr and sulfonated groups by the application of constant voltage to reaction medium. In the presence of electrolyte, under certain voltage $(2.5 \mathrm{~V})$, functionalization of solid particles became more obvious in FTIR spectrum results. Therefore, change in the selectivity values of degradation products were conducted with the functionalization of MCC particles due to applied voltage under sub-critical conditions.

Keywords Cellulose $\cdot$ Sub-critical water . Electrochemical $\cdot$ Levulinic acid · 5-HMF

\section{Introduction}

Biomass decomposition products such as Levulinic acid (LVA), Hydroxymethyl furfural (5-HMF) and Furfural have wide range of potential derived compounds that can be used in different areas such as gasoline additive, polymers etc.

Due to its potential, biomass has been investigated widely in both academia and industry in order to find more efficient and sustainable way of utilization.

In this purpose, different methods in utilization of biomass have been reported so far; mineral acid ( $\mathrm{Li}$ et al. 2017), ionic liquids (Zheng et al. 2016), solid acid catalysts (Kilic and Yilmaz 2015), sub- and supercritical water (Sasaki et al. 2000). Electrochemical methods have also been reported, Zhang and coworkers studied electrochemical degradation of lignin (Zhang et al. 2014), Xu and coworkers studied 
electrolysis of corn stover to sugars (Xu et al. 2014). Most of the electrochemical reactions reported in literature carried out in ambient conditions. Sub- and supercritical water as reaction medium have attracted attention due to its versatile properties such as ionic product concentration $\left(10^{-11}, 200-300{ }^{\circ} \mathrm{C}\right.$ range $)$ and dielectric constant $(\varepsilon \approx 10$ near critical) (Marshall and Franck 1981). Self-dissociation products of water as hydroxide $\left(\mathrm{OH}^{-}\right)$and hydronium ion $\left(\mathrm{H}_{3} \mathrm{O}^{+}\right)$play crucial role in the protonation of $\beta$-glycosidic bond of cellulose that resulted in formation of decomposition products. In order to depolymerize microcrystalline cellulose, hydronium ion must access to intra- and inter-molecular hydrogen bonds. Thus, diffusion of protons with versatile properties (low density, high diffusivity) of near critical water becomes more effective. High ionic product concentration also yields to low energy for the migration of electroactive species in electrochemical methods. Therefore, electrochemical reactions at near critical conditions become more economically feasible (Asghari and Yoshida 2008).

As hybrid method of electrochemical oxidation and synthesis of organic compounds (Sasaki et al. 2007) in sub-critical water conditions become attractive for the production of value added chemicals (Yuksel et al. 2011). In our previous study, we investigated the decomposition of microcrystalline cellulose via application of direct constant current (0-2 A) in sub-critical water conditions $\left(170-230^{\circ} \mathrm{C}\right)$ with the addition of sulfuric acid (1-50 mM). In order to reveal the coupled and interaction parameters of constant current with sulfuric acid under hydrothermal conditions, fractional factorial experimental design was built. It was found that, application of $1 \mathrm{~A}$ current to reaction medium decreased required temperature from 230 to $200{ }^{\circ} \mathrm{C}$ to reach maximum cellulose conversion of $82 \%$ with the presence of $50 \mathrm{mM} \mathrm{H}_{2} \mathrm{SO}_{4}$ (Akin and Yuksel 2016). Increase in TOC amount was conducted with the formation of ionic products $\left(\mathrm{OH}^{-}, \mathrm{H}_{3} \mathrm{O}^{+}\right)$and radicals $\left(\mathrm{OH}^{-}, \mathrm{SO}_{4}^{-}\right)$due to the applied direct current. Higher current value $(2 \mathrm{~A})$ resulted in the decreased TOC showing that gaseous products were formed due to the high concentration of radicals in the reaction medium. Effect of current on cellulose decomposition products such as 5-HMF and levulinic acid was also investigated and found that direct current (1 and $2 \mathrm{~A}$ ) increased yield of 5-HMF in contrast to levulinic acid concentration. Applied constant current to reaction medium resulted in the variable electrode potential throughout the reaction. Thus, formation of activated species within the reaction medium varies as the electrode potentials changes. Davis and coworkers studied the formation of persulfate via electrolysis of sulfuric acid solution based on radical mechanism (Davis et al. 2014). They reported that different radical species $\left(\mathrm{SO}_{4}^{-}, \mathrm{HSO}_{4}^{\bullet}, \mathrm{OH}^{\bullet}\right)$ and ionic products $\left(\mathrm{SO}_{4}{ }^{2-}, \mathrm{H}_{3-}\right.$ $\mathrm{O}^{+}, \mathrm{OH}^{-}$) can be formed during electrolysis of sulfuric acid solution at different electrode potentials. Applied constant voltage can form activated species as radicals (Fig. 1b) and ionic products (Fig. 1a) of sulfuric acid and water in sub-critical water. Formation of these products can alter the decomposition mechanism (Fig. 1) of cellulose in a selective way of degradation products such as levulinic acid, furfural and 5-HMF. Radical based decomposition mechanism of glucose is favored in supercritical water conditions (Promdej and Matsumura 2011) and cellobiose decomposition mechanism in sub- and supercritical conditions was also reported in details (Kabyemela et al. 1998). They reported formation of glucosyl-glycoaldehyde, glucosyl-eryhtrose and glycoaldehyde was observed under supercritical water conditions. As the application of constant voltage resulted in formation of ionic and radical species, decomposition mechanism of cellulose via ionic and radical based pathways is postulated (Fig. 2)

In this study, decomposition of microcrystalline cellulose via the application of constant voltage in subcritical conditions $\left(200{ }^{\circ} \mathrm{C}\right)$ with and without sulfuric acid (5-25 mM) addition was investigated. Cellulose decomposition was conducted with TOC measurements and effect of applied voltage (2.5, 4.0 and $8.0 \mathrm{~V}$ ) on product distribution was investigated by selectivity and yield values.

\section{Materials and methods}

Microcrystalline cellulose (MCC, Sigma Aldrich, particle size $<20 \mu \mathrm{m}$ ) was used as model compound. High Performance Liquid Chromatogram (HPLC, Agilent 1100) analysis was used for quantitative analysis of chemicals as glucose, fructose, 5-HMF, levulinic acid, and furfural. Separation of products were maintained via sugar column (Shodex, SH1100) with elution of sulfuric acid (Merck, 96-98\% purity) at $3.75 \mathrm{mM}$ with the flow rate of $0.5 \mathrm{ml} \mathrm{m^{-1 }}$ at a 
(a)

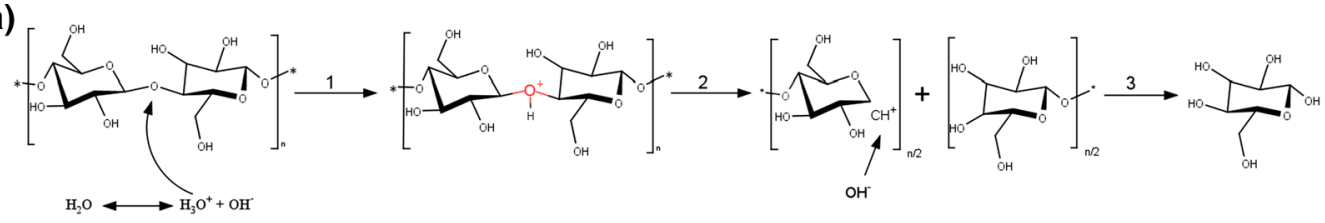

(b)

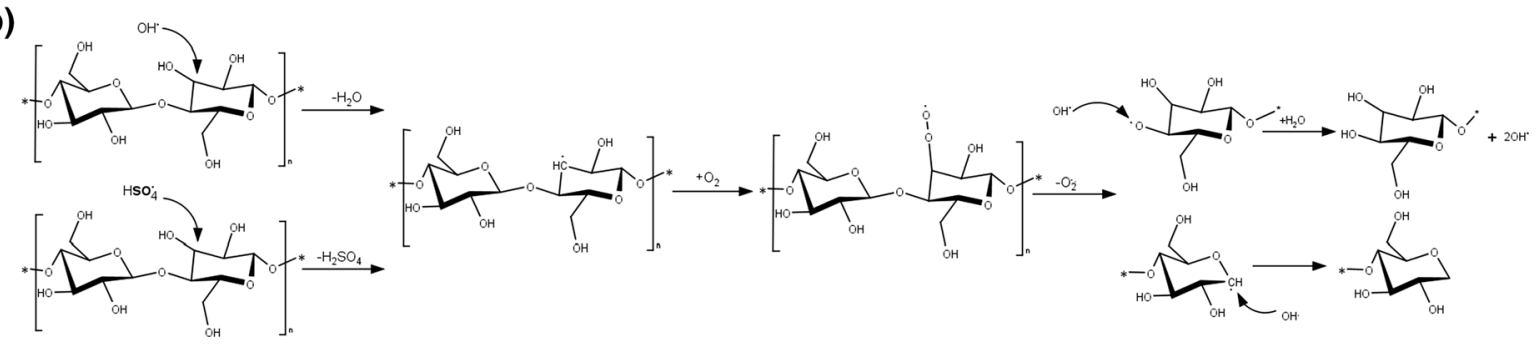

Fig. 1 Suggested mechanism of cellulose decomposition by $\mathbf{a}$ ionic product and $\mathbf{b}$ radical based pathway

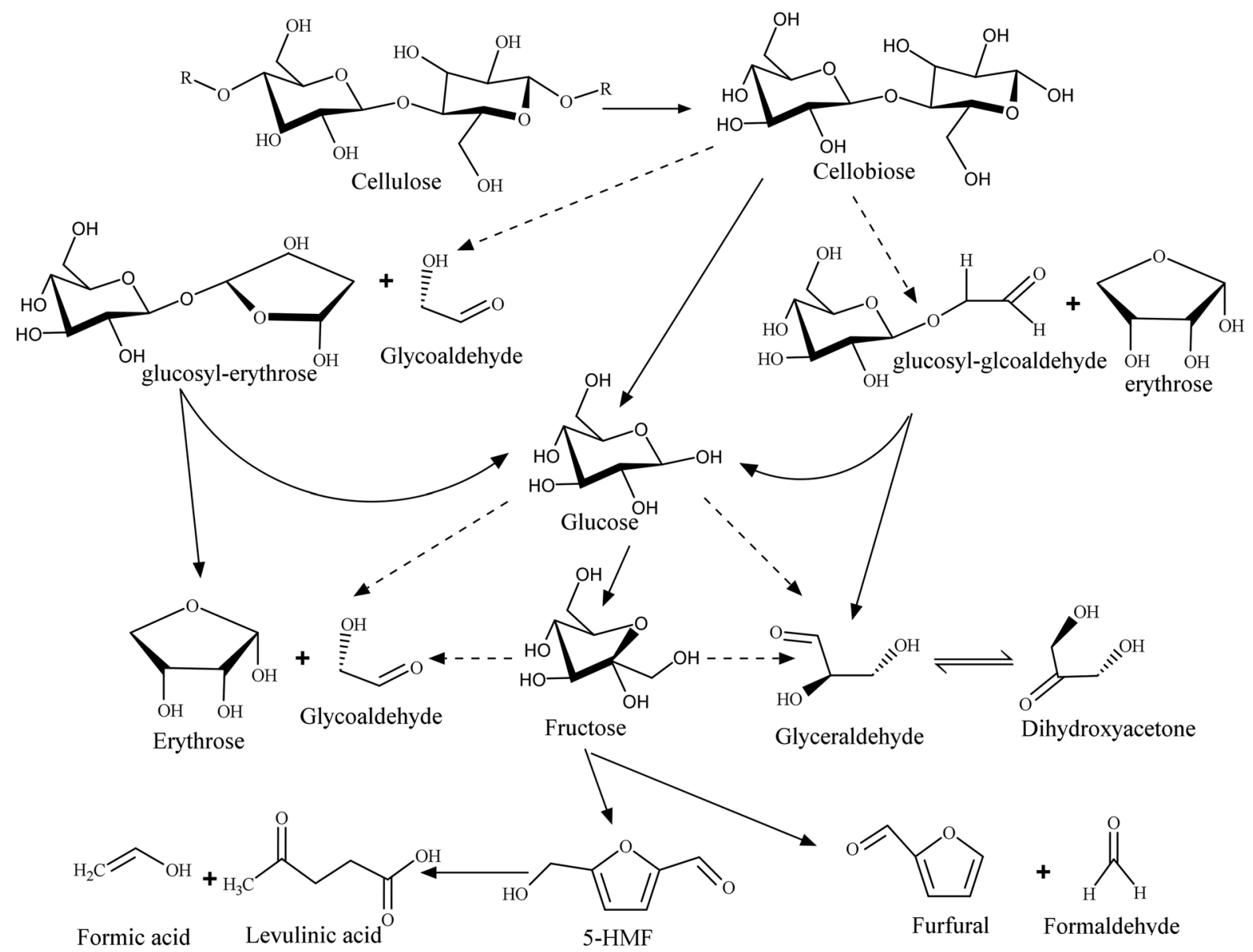

Fig. 2 Postulated reaction pathways of cellulose decomposition by radical (dashed line arrow) and ionic (solid line arrow) species based mechanism 
column temperature of $50{ }^{\circ} \mathrm{C}$. Aldehydes, ketones and carboxylic acids were identified by refractive index (RI) detector. Gaseous product was collected by polypropylene (PP) bags at the end of reaction. Gas Chromatography equipped with a Thermal Conductivity Detector (GC-TCD) (Agilent $6890 \mathrm{~N}$ ) were used to analyze gas products. TOC analyzer (Shimadzu TOC-VCPH) was used to monitor TOC conversions in the liquid products and solid residues. Fourier Trans- quantified via HPLC. Yield (Eq. 1) and selectivity (Eq. 2) values of degradation products were calculated based on the carbon balances with the following equations. Initial carbon amount loaded to the reaction medium was analyzed via TOC and it was found that $42-45 \%$ of MCC is composed of carbon. Hence, yield calculations based on initial moles of carbon content was conducted as $0.28 \mathrm{~mol}(8 \mathrm{~g}$ of $\mathrm{MCC})$ of initial carbon amount.

Yield $\%=\frac{(\# \text { of carbon of species }) \times(\text { Mole of species produced })}{\text { Moles of carbon in cellulose }} \times 100$

Selectivity $\%=\frac{(\# \text { of carbon of species }) \times(\text { Mole of species produced })}{\text { Total Moles of Carbon }(\text { TOC }) \text { in liquid }} \times 100$

mission Infrared Spectroscopy (FT-IR) analyses of solid residues were conducted by Perkin Elmer Frontier-Spectra two with scan rate of $4 \mathrm{~cm}^{-1}$ from $600 \mathrm{~cm}$ to $4000 \mathrm{~cm}^{-1}$ wavenumber

Electrochemical degradation of MCC under hydrothermal conditions were carried out in a $450 \mathrm{ml}$ (Parr 5500 series) of batch reactor equipped with a specially designed titanium anode. Experimental setup was illustrated and explained in detail at our pervious study (Akin and Yuksel 2016). Sulfuric acid at different concentration $(0,5$ and $25 \mathrm{mM})$ was added to reaction medium as external proton source. Hydrothermal electrolysis experiments were carried out at a constant temperature of $200{ }^{\circ} \mathrm{C}$ for $240 \mathrm{~min}$ of reaction time by application of constant voltage values $(2.5,4.0$ and $8.0 \mathrm{~V})$. Constant potential difference across anode (titanium) and reactor wall (T316) was supplied by DC power source (GW Instekt). Liquid samples were collected during reaction for $30 \mathrm{~min}$ ' intervals and analyzed by HPLC and TOC instruments.

\section{Results and discussion}

TOC and product distribution

Cellulose degradation products such as glucose, fructose, levulinic acid, 5-HMF and furfural were
Ionic product concentration plays crucial role in destruction of intra- and inter hydrogen bonds of cellulose that yields the formation of further degradation products. Cellulose decomposition mechanism in sub-critical conditions is explained via ionic based reactions (Sasaki et al. 2000). Our previous study showed that application of constant current (1 A) to reaction medium resulted in the highest TOC yield in comparison to $2 \mathrm{~A}$ and current free reactions. An increase in ionic concentration due to self-dissociation of water under applied current resulted in higher TOC yields, in comparison to higher current values (2 A) that yields the formation of gaseous products due to radical based mechanism in decomposition of cellulose (Akin and Yuksel 2016).

In order to observe the effect of applied constant voltage to ionic product concentration in reaction medium, TOC values were investigated (Fig. 3).

When cellulose degradation was carried out under subcritical conditions without electrolyte and applied voltage, TOC yielded to $13 \%$ (Fig. 3a). In the absence of electrolyte $\left(\mathrm{H}_{2} \mathrm{SO}_{4}\right)$, application of constant voltage $(2.5$ and $4.0 \mathrm{~V}$ ) resulted in lower TOC values (Fig. 3a) in comparison to current free experiment. Lower TOC value could be resulted due to decrease in ionic product concentration because of applied voltage. In the absence of electrolyte, hydronium $\left(\mathrm{H}_{3} \mathrm{O}^{+}\right)$and hydroxyl $\left(\mathrm{OH}^{-}\right)$ions could be reduced or oxidized in 

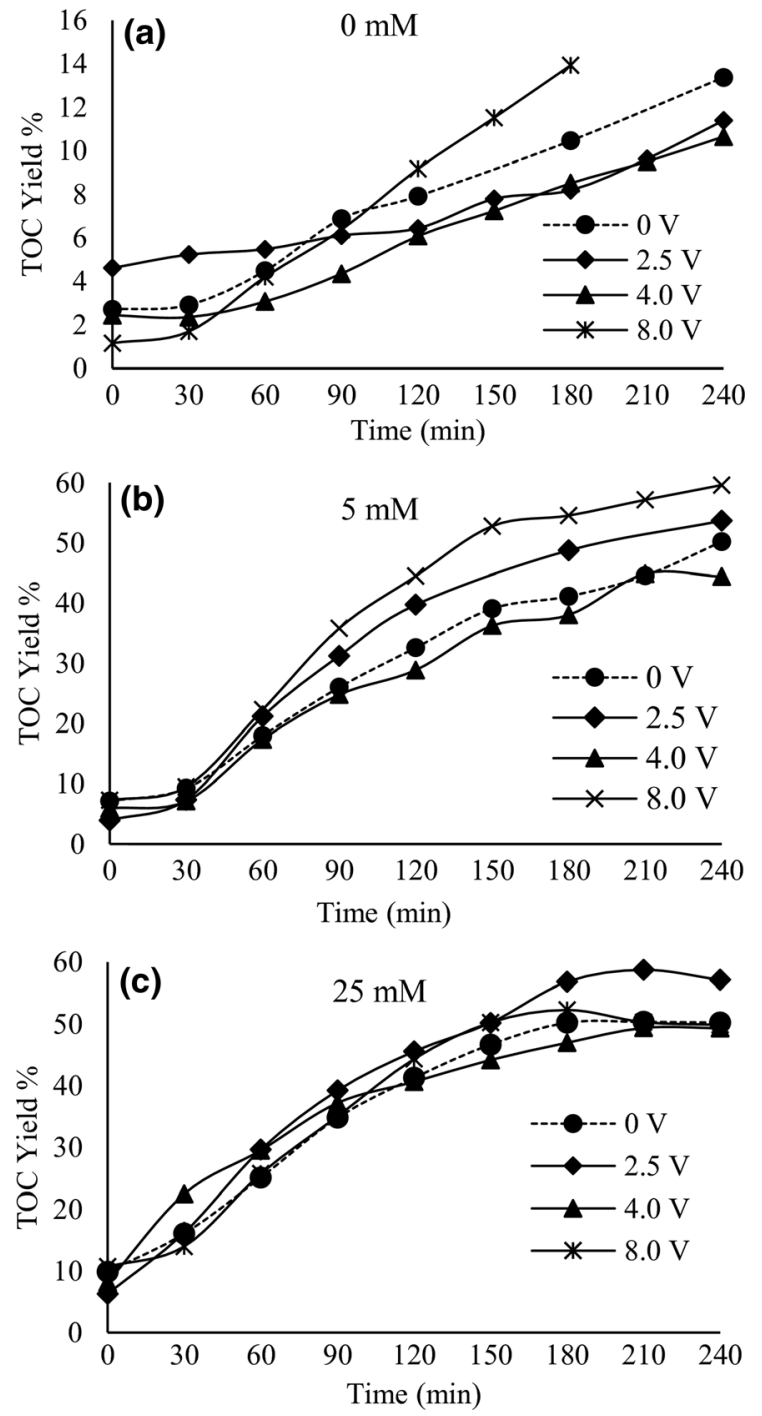

Fig. 3 Total organic content (TOC) of liquid products at applied voltage values of $0,2.5,4.0,8.0 \mathrm{~V}$ at different electrolyte $\left(\mathrm{H}_{2} \mathrm{SO}_{4}\right)$ concentrations; a $0 \mathrm{mM}$, b $5 \mathrm{mM}$, c $25 \mathrm{mM}$

cathode and anode respectively. Thus, protonation of $\beta$-gycosidic bond could be limited due to the low concentration of ion products. However, application of $8.0 \mathrm{~V}$ of potential difference resulted in higher TOC value (Fig. 3a). This could be explained by overpotential that resulted in the dissociation of water to its ionic products. Application of voltage under hydrothermal conditions also yields the formation of radical species such as hydroxyl radical $\left(\mathrm{OH}^{\bullet-}\right)$ (Rossmeisl et al. 2005). Increase in radical species concentration could also result in the formation of decomposition products via radical based mechanism that yields to formation of glucosyl-glycoaldehyde, glucosyl-eryhtrose (Fig. 2). Addition of sulfuric acid $(5 \mathrm{mM})$ to the reaction medium increased the TOC yield to $50 \%$ (Fig. 3b) under hydrothermal conditions. Application of 2.5 and $8.0 \mathrm{~V}$ voltages to reaction medium containing $5 \mathrm{mM}$ of sulfuric acid increased the TOC yield to 54 and $60 \%$, respectively. There is coupled effect of applied voltage and sulfuric acid in TOC yield as reported in our previous study (Akin and Yuksel 2016). The coupled effect can be explained by the oxidation of sulfuric acid that yields the formation of sulfate radical $\left(\mathrm{SO}_{4}^{--}\right)$near anode (Davis et al. 2014). However, application of $4.0 \mathrm{~V}$ did not alter TOC yield. The interaction of applied voltage with the parameters effecting TOC yield is indistinguishable. It is due to the fact that TOC formation is also effected by the formation of carboxylic acids that are formed during the decomposition of cellulose. Addition of sulfuric acid increases the concentration of degradation products, hence, effect of applied voltage at higher sulfuric acid concentration showed different patterns in TOC yield (Fig. 3c). Applied $2.5 \mathrm{~V}$ resulted in the higher TOC yield in reaction medium with $25 \mathrm{mM}$ of sulfuric acid.

\section{Product yield and selectivity}

Effect of applied voltage $(2.5,4.0$ and $8.0 \mathrm{~V})$ on product yield and selectivity values in hydrothermal reaction medium was investigated with and without acid catalyst $\left(\mathrm{H}_{2} \mathrm{SO}_{4}\right)$. Yield and selectivity values of cellulose degradation products such as glucose, fructose, furfural, 5-HMF and levulinic acid were analyzed and quantified as described in Materials and methods section. The control reaction was conducted under hydrothermal conditions $\left(200{ }^{\circ} \mathrm{C}\right)$ without acid and voltage application to the reaction medium. Under hydrothermal conditions, selectivity (Fig. 4a) and yield (Fig. 4b) values of glucose reached to 15 and $6 \%$, respectively. Application of voltage (2.5 and $4.0 \mathrm{~V}$ ) decreased the yield of glucose below $2 \%$. This could be due to same effect of voltage as it was in TOC yield. In the absence of electrolyte, ion products of water can be involved redox reactions that can hinder the protonation of cellulose. However, application of $8.0 \mathrm{~V}$ resulted in higher selectivity to glucose (Fig. 4a) in comparison to 2.5, 4.0 V and voltage free experiments. The same trend was also observed in glucose isomerization to fructose (Fig. 4c, d). 
Fig. 4 Selectivity and yield values of degradation products $\mathbf{a}-\mathbf{b}$ glucose, $\mathbf{c}-\mathbf{d}$ fructose, e-f furfural, $\mathbf{g}-\mathbf{h}$ 5-HMF, i-j levulinic acid by hydrothermal electrolysis without acid catalyst (a)

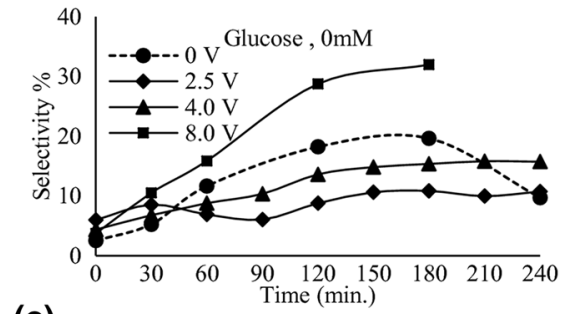

(c)
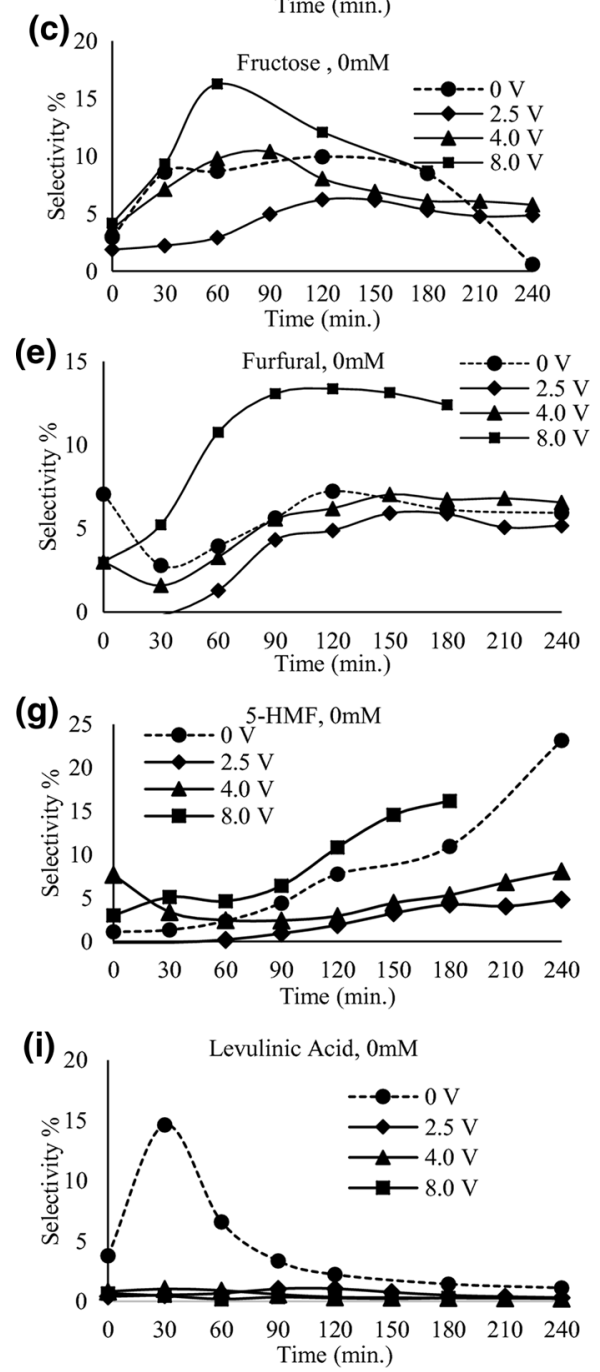

(b)
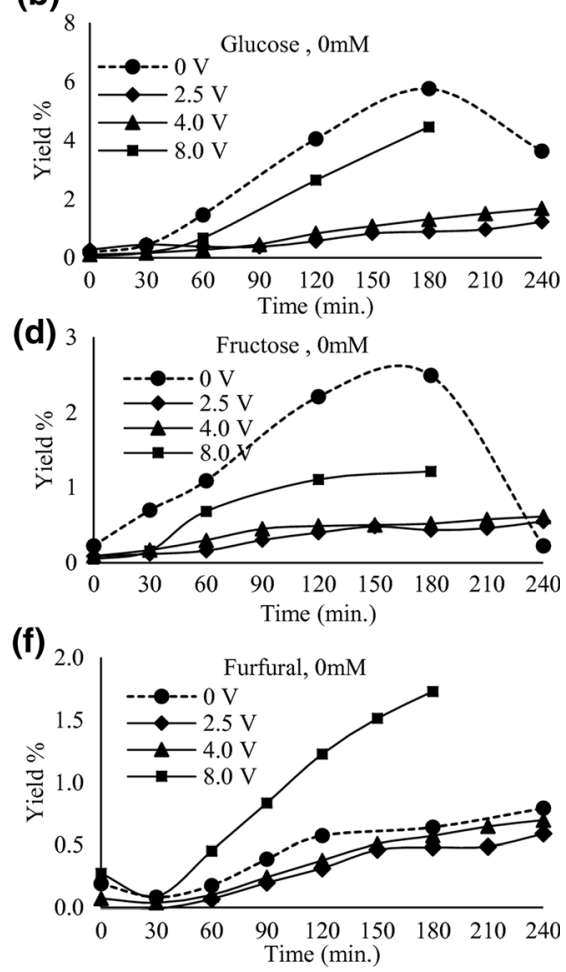

(h) 4
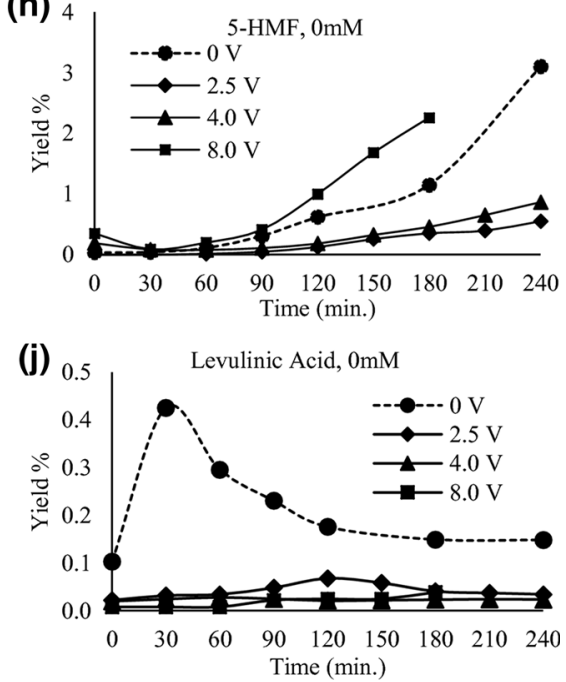

However, under hydrothermal conditions, yield of fructose was much higher than that was in voltage applied experiments. This could be explained by series reactions in which fructose was further converted to 5-HMF by dehydration.

Applied voltage $(8.0 \mathrm{~V})$ shifted the selectivity in favor of furfural (Fig. 4e) and 5-HMF (Fig. 4g) formation. There are two proposed reaction pathways for the conversion of fructose to furfural under hydrothermal conditions (Aida et al. 2007). One is the removal of formaldehyde from 5-HMF and the other one is the cleavage of $\mathrm{C}-\mathrm{C}$ bond in fructose that yields pentose formation and further dehydration of pentose yields to furfural formation (Luijkx et al. 1993). The yield values of furfural (Fig. 4f) and 5-HMF (Fig. 4h) increased simultaneously. 
Therefore, it could be said that furfural might be formed by the cleavage of $\mathrm{C}-\mathrm{C}$ bond in fructose because of the low yield of fructose $(8.0 \mathrm{~V})$ in comparison to hydrothermal reaction $(0 \mathrm{~V})$ (Fig. $4 \mathrm{~d})$. Otherwise, 5-HMF concentration was expected to decrease with the increase of furfural yield in the reaction pathway of formaldehyde extraction from 5-HMF. Rehydration of 5-HMF resulted in formation of levulinic acid (Fig. 4i). Application of voltage limited the formation of levulinic acid as compared to the voltage free experiments.

In our previous work, effect of addition of external proton source $\left(\mathrm{H}_{2} \mathrm{SO}_{4}\right)$ on product yields at molarity of 25 and $50 \mathrm{mM}$ under hydrothermal electrolysis (constant current) conditions has already been investigated (Akin and Yuksel 2016). Statistical analysis showed that there was a coupled effect of electrolysis and acid catalyst on TOC yield under hydrothermal conditions. However, constant current did not selectively alter the decomposition reaction. In this study, it was proposed that application of constant voltage may alter the decomposition of cellulose in a selective way. Thus, yield and selectivity values of decomposition products under applied constant voltage of 2.5, 4.0 and $8.0 \mathrm{~V}$ were investigated with addition of $5 \mathrm{mM}$ (Fig. 5) and $25 \mathrm{mM}$ (Fig. 6) acids into reaction medium.

Addition of electrolyte distinguished the diverse effect of applied voltage on glucose (Fig. 5b) and fructose (Fig. 5d) yields as it was in the case of electrolyte free experiment $(0 \mathrm{mM})$. Hydrothermal electrolysis products selectively produced glucose (Fig. 5a) and fructose (Fig. 5c) at first $30 \mathrm{~min}$ of reaction. Glucose selectivity reached to $17.9 \%$ under hydrothermal conditions within $30 \mathrm{~min}$ without applied voltage. Application of $2.5 \mathrm{~V}$ increased glucose selectivity to $45 \%$ at which TOC conversions were about $10 \%$ for both conditions. It is an evident that rate of protonation of $\beta$-glyosidic bond of cellulose increased under certain potential voltage $(2.5 \mathrm{~V})$ and that yielded higher glucose selectivity. Selective increase in the glucose selectivity within $30 \mathrm{~min}$ could be due to the both increase in both ionic and radical species concentration as in the suggested decomposition mechanism (Fig. 2).

At higher voltage $(4.0$ and $8.0 \mathrm{~V})$, glucose selectivity was lower than voltage free $(0 \mathrm{~V})$ experiment at similar TOC (Fig. 3b) values. Similar TOC indicate that partial decomposition of cellulose yielded oligomers at higher voltage values within $30 \mathrm{~min}$. High potential differences resulted in the formation of hydrogen gases instead of protonation of $\beta$-glyosidic bond of cellulose as found in GC-TCD analysis (Fig. 7a) of gaseous products. Moreover, our previous study also showed consistent results in terms of gaseous products formation at higher current values (Akin and Yuksel 2016).

The selectivity values of degradation products such as 5-HMF (Fig. 5g) and levulinic acid (Fig. 5i) dramatically changed after $30 \mathrm{~min}$ of reaction. 5HMF selectivity sharply increased up to $28 \%$ between 30 and $90 \mathrm{~min}$ of reaction time. The maximum selectivity value of 5-HMF $(29 \%)$ is reached at $2.5 \mathrm{~V}$ within $120 \mathrm{~min}$ of reaction time. Under subcritical water conditions $(0 \mathrm{~V}), 5$-HMF rehydrated to levulinic acid due to the thermal instability, which was also reported in literature (Sasaki et al. 2011). Therefore, levulinic acid was formed via rehydration of 5-HMF and its selectivity reached to $21 \%$ at $2.5 \mathrm{~V}$ within $240 \mathrm{~min}$ of reaction. The dramatic change in 5-HMF selectivity under applied 2.5 V could be due to the functionalization of cellulose surface with carboxylic acid and sulfoxide groups. It was reported that Bronsted acid sites play crucial role in selective formation of 5-HMF via dehydration of fructose (Qi et al. 2012; Swift et al. 2014). Thus, the dramatic change in 5-HMF and levulinic acid selectivity values at $2.5 \mathrm{~V}$ potential could be due to the formation of sulfonated carbon particles that can act as catalyst in selective formation of 5-HMF. Formation of sulfone groups were detected via FTIR (Fig. 9a) of remaining solid particles at the end of reaction. FTIR patterns showed that formation of sulfur ester $(\mathrm{S}-\mathrm{R})$ and sulfoxide $(S=O)$ groups became more obvious at $2.5 \mathrm{~V}$ of applied voltage. The detailed discussion is held on spectroscopic analysis part. Moreover, sulfonated carbon derived from biomass has been investigated and reported in selective production of 5-HMF (Kang et al. 2013; Qi et al. 2012; Shen et al. 2016). However, application of $4.0 \mathrm{~V}$ and $8.0 \mathrm{~V}$ of potential difference did not alter the selectivity values of 5-HMF and levulinic acid. The formation of carboxylic and sulfonated functional groups in solid residue was also observed at $8.0 \mathrm{~V}$ potential. However, catalytic effect of functional groups was not observed. In addition, application of $8.0 \mathrm{~V}$ decreased the product selectivities in contrast to TOC yields. Application of higher voltage $(8.0 \mathrm{~V})$ decreased the $\mathrm{pH}$ value to 1.72 that was 2.0 at voltage free 
Fig. 5 Selectivity and yield values of degradation products $\mathbf{a}-\mathbf{b}$ glucose, $\mathbf{c}-\mathbf{d}$ fructose, e-f furfural, $\mathbf{g}-\mathbf{h}$ 5-HMF, $\mathbf{i}-\mathbf{j}$ levulinic acid by hydrothermal electrolysis with $5 \mathrm{mM}$ sulfuric acid catalyst (a)

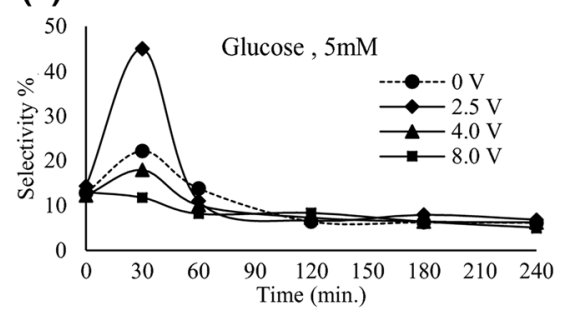

(c)

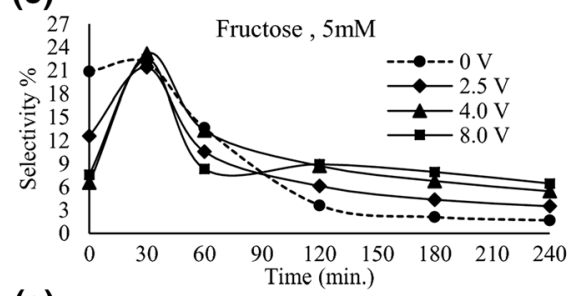

(e)

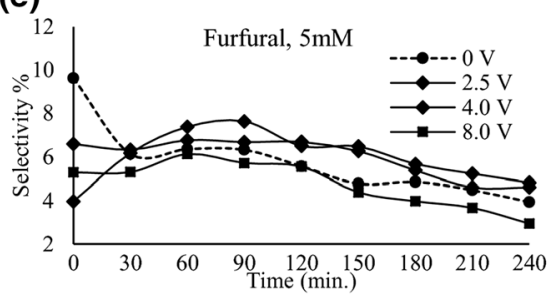

(g)
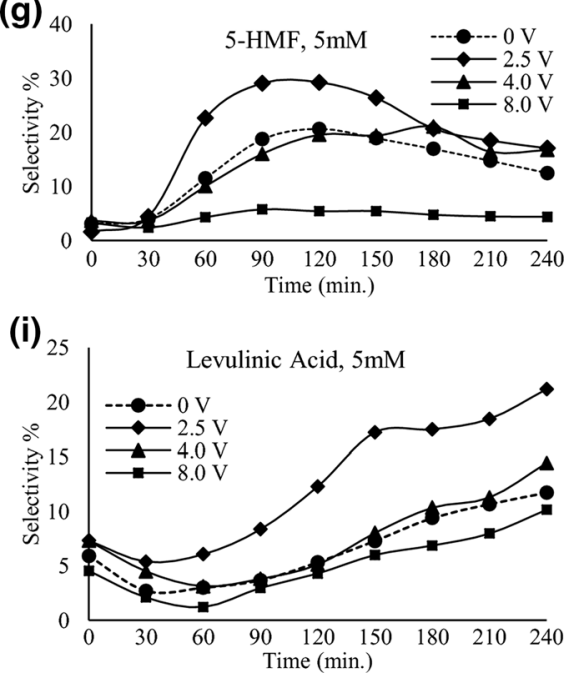

(b)

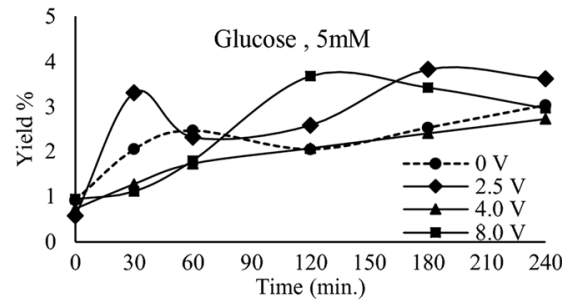

(d)

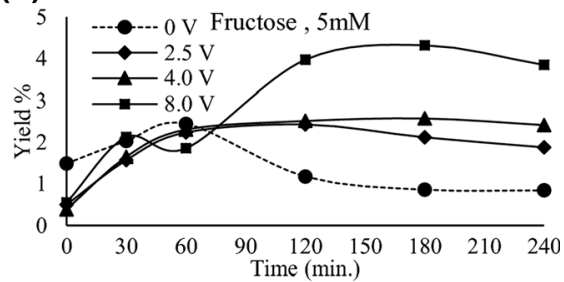

(f)

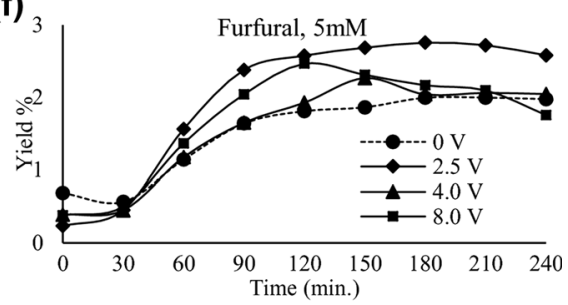

(h)

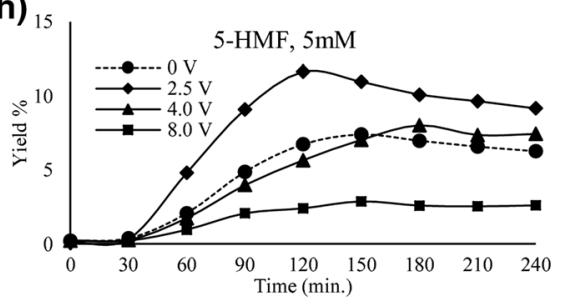

(j)

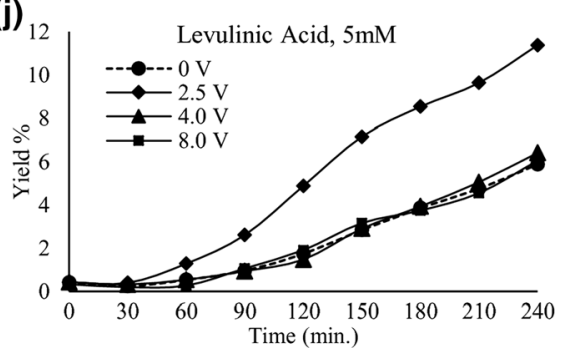

experiment. Applied higher voltage could have resulted in further degradation of aldehydes and ketones to carboxylic acids due to the formation of highly active sulfate ion radicals resulted in the fragmentation reactions. It was reported that levulinic acid also involved further kolbe reactions that yields the formation of valeric acid (dos Santos et al. 2015). Thus, under applied voltage of $8.0 \mathrm{~V}$ based on the
TOC results, levulinic acid could further involved the formation degradation products.

Effect of applied voltage at higher acid concentration $(25 \mathrm{mM})$ on product yield and selectivity values was given in Fig. 6. Under hydrothermal conditions, selectivity of 5-HMF (Fig. 6g) reached to $40 \%$ within $60 \mathrm{~min}$ and further reaction time (210 min) yielded levulinic acid (Fig. 6i) with 
(a)

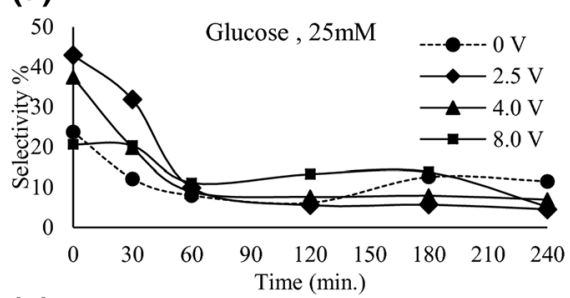

(c)

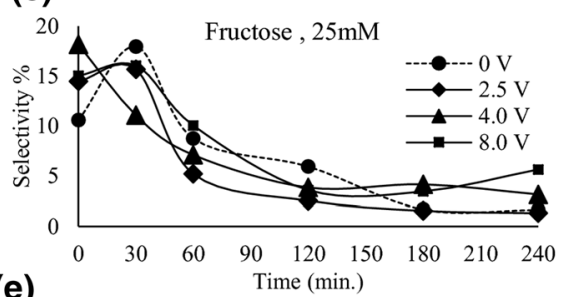

(e)

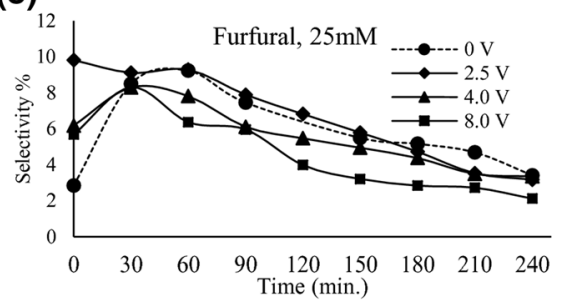

(g)
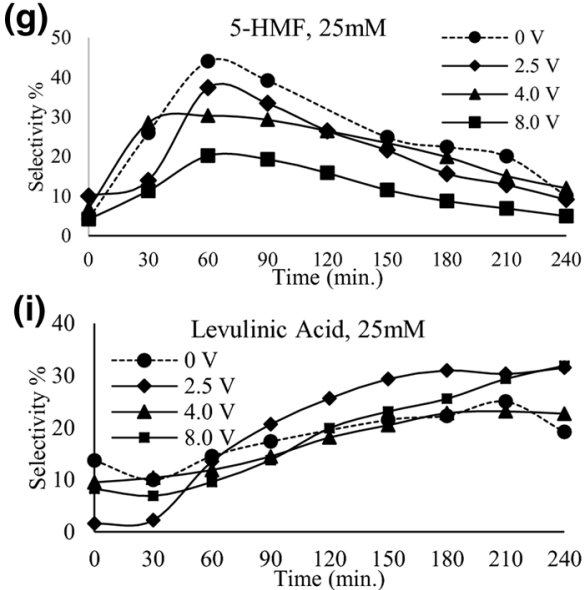

(b)

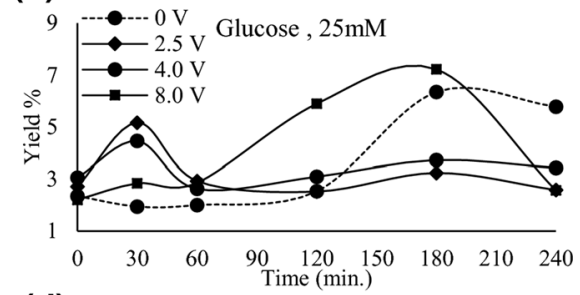

(d)
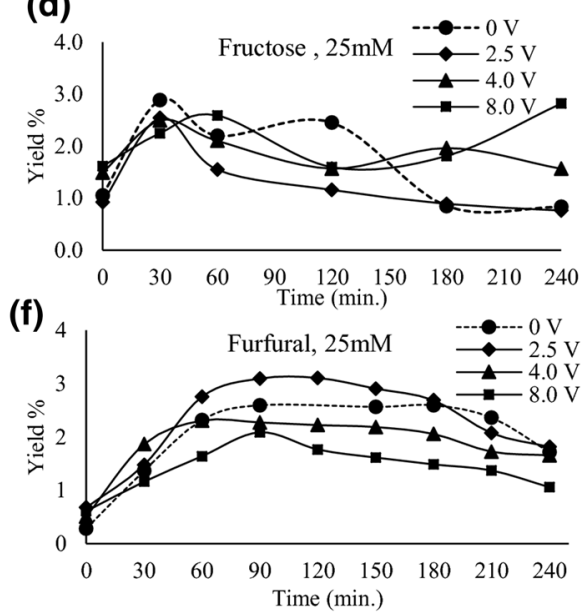

(h)
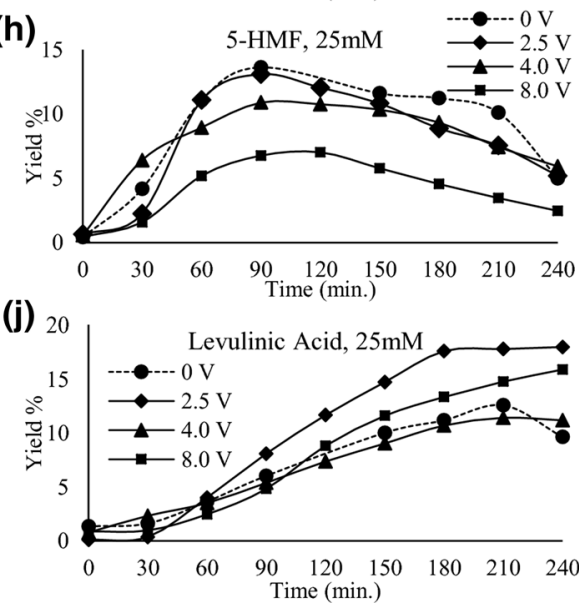

Fig. 6 Selectivity and yield values of degradation products $\mathbf{a}-\mathbf{b}$ glucose, $\mathbf{c}-\mathbf{d}$ fructose, $\mathbf{e}-\mathbf{f}$ furfural, $\mathbf{g}-\mathbf{h}$ 5-HMF, i-j levulinic acid by hydrothermal electrolysis with $25 \mathrm{mM}$ sulfuric acid catalyst

selectivity of $23 \%$. Application of voltage (4.0 and $8.0 \mathrm{~V}$ ) showed diverse effect on 5-HMF selectivity even though the FTIR results showed that sulfone groups were formed at solid residuals. Application of potential at relatively high concentration of sulfuric acid may yield high concentrations of reactive species such as sulfone ion radicals that hinders the effect of sulfonated carbon particles. In contrast, levulinic acid selectivity increased from $23 \%(0 \mathrm{~V})$ to $32 \%$ (2.5 and
$8.0 \mathrm{~V}$ ) by application of voltage. GC-TCD (Fig. 7c) analysis showed that under hydrothermal conditions there was no significant production of gaseous products, however, as the potential applied, carbon monoxide and carbon dioxide formation increased. Thus, application of voltage under $25 \mathrm{mM}$ acid yielded the formation of gaseous product due to further decomposition based on radical mechanism (Sasaki et al. 2004). 
(a)

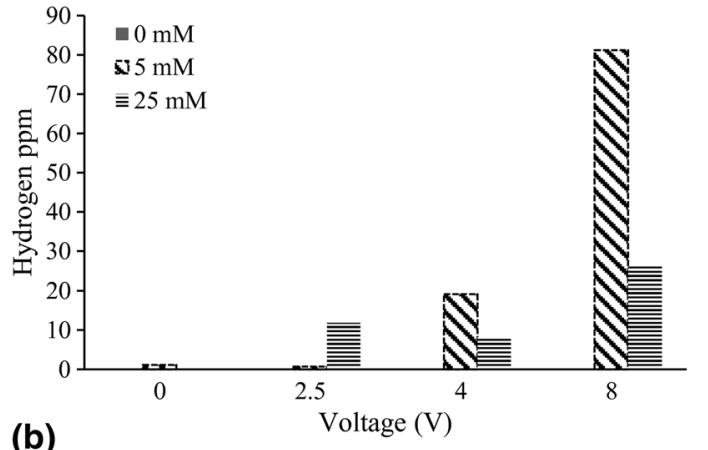

(b)
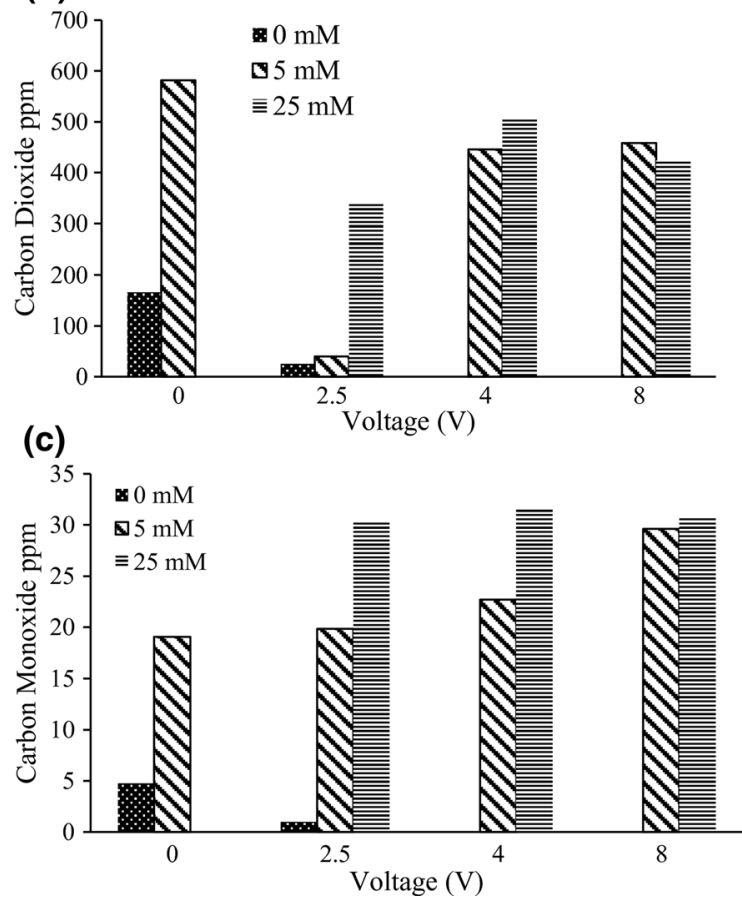

Fig. 7 Formation of gaseous products of $\mathbf{a}$ hydrogen, $\mathbf{b}$ carbon dioxide, $\mathbf{c}$ carbon monoxide under acid catalyst $(5,25 \mathrm{mM})$ and hydrothermal conditions with applied voltage of $2.5,4.0$ and $8.0 \mathrm{~V}$

\section{Gas products formation}

Gas samples were collected at the end of reaction (240 min) into PP bags and analyzed by GC-TCD. Hydrolysis of cellulose first yielded to aldehydes and ketones and further decomposition resulted in formation of carboxylic acids that could be decomposed to the gases under hydrothermal conditions. Application of potential difference to the reaction medium resulted in the formation of hydrogen and oxygen gases because of reduction and oxidation reactions of ionic products of water. Moreover, addition of sulfuric acid resulted in the formation of radical species that end-up formation of gaseous products due to radical based decomposition mechanism. Formation of gas products such as hydrogen (Fig. 7a), carbon monoxide (Fig. 7b) and carbon dioxide (Fig. 7c) were investigated. Application of potential of $2.5 \mathrm{~V}$ decreased carbon dioxide (Fig. 7b) formation in electrolyte free and $5 \mathrm{mM}$ sulfuric acid containing reaction mediums. Decrease in the carbon dioxide could be explained by the electrochemical reduction at cathode surface (Jitaru et al. 1997) and possible further reactions with aldehydes and ketones. Further increase in voltage resulted in higher concentration of gaseous product. Moreover, when $8.0 \mathrm{~V}$ of potential was applied, methane was produced in the presence of 5 and $25 \mathrm{mM}$ sulfuric acid. Electrochemical reduction of carbon dioxide might result in the formation of methane (Fig. 8).

\section{FTIR spectrum of solid residues}

The FTIR spectrums of solid residues of acid free (Fig. 10) experiments and with $5 \mathrm{mM} \quad \mathrm{H}_{2} \mathrm{SO}_{4}$ hydrothermal electrolysis reactions (Fig. 9) were investigated. Raw material microcrystalline cellulose showed the stretching vibrations at 3341 and $2877 \mathrm{~cm}^{-1}$ attributed to $\mathrm{O}-\mathrm{H}$ and $\mathrm{C}-\mathrm{H}$ bonds. In addition, the peak at $902 \mathrm{~cm}^{-1}$ is attributed to the $\beta$-Dglucopyranosyl that disappeared by the application of $8.0 \mathrm{~V}$ of potential under acidic conditions (Fig. 6b). In contrast, application of $4.0 \mathrm{~V}$ did not totally diminish the intermolecular hydrogen bond peak, however, $2.5 \mathrm{~V}$ application was almost destruct the $\beta$-D-glucopyranosyl. The main characteristic peaks of cellulose were observed at spectrum of 1033 and $1059 \mathrm{~cm}^{-1}$ and were assigned as $\mathrm{C}-\mathrm{OH}$ and $\mathrm{C}-\mathrm{O}$ stretching vibrations, respectively. The peaks of microcrystalline cellulose at 1104 and $1160 \mathrm{~cm}^{-1}$ could be attributed to $\mathrm{C}-\mathrm{O}$ and $\mathrm{C}-\mathrm{C}$ stretching vibration of cellulose ether. Application of voltage in sulfuric acid reaction medium yielded the formation of sulfonate functionalities at solid residues. The peak of sulfur ester ( $\mathrm{S}-$ $\mathrm{OR})$ and sulfoxide $(\mathrm{S}=\mathrm{O})$ were observed at wavenumber of $811 \mathrm{~cm}^{-1}$ and $1030-1060 \mathrm{~cm}^{-1}$, respectively. The sulfoxide peaks were overlapped with the characteristic peaks of cellulose and it was hard to distinguish. However, the shoulder (Fig. 9, arrow) at $1033 \mathrm{~cm}^{-1}$ of cellulose was separated at spectrums of 2.5 and $8.0 \mathrm{~V}$. Moreover, the relative 


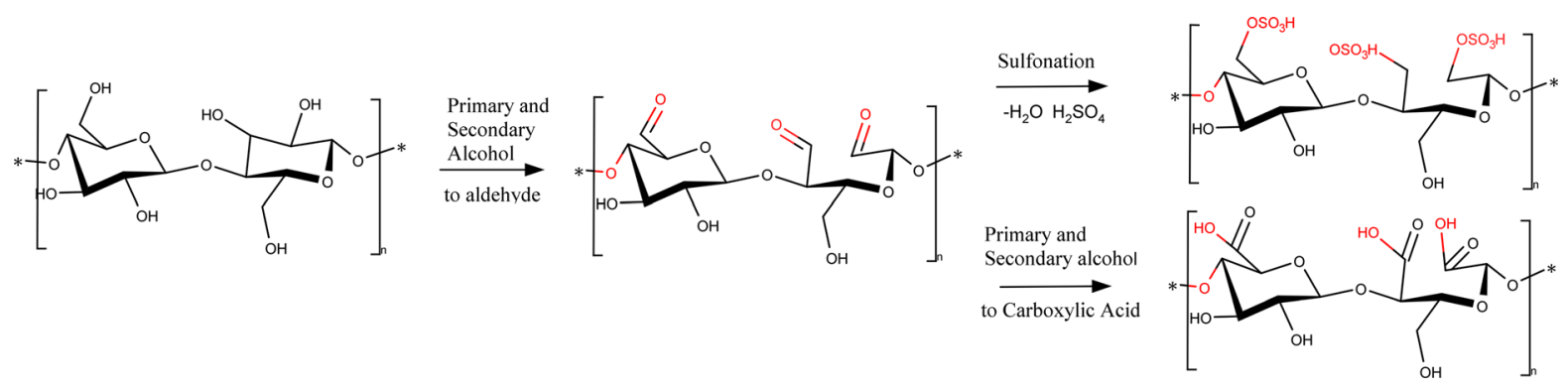

Fig. 8 Reaction mechanism of formation of carboxylic acid and sulfoxide functional groups by oxidation of primary and secondary alcohol group of cellulose

Fig. 9 FTIR spectrum of microcrystalline cellulose and solid residues of applied potential of $2.5,4.0$ and $8.0 \mathrm{~V}$ in $5 \mathrm{mM}$ sulfuric acid medium

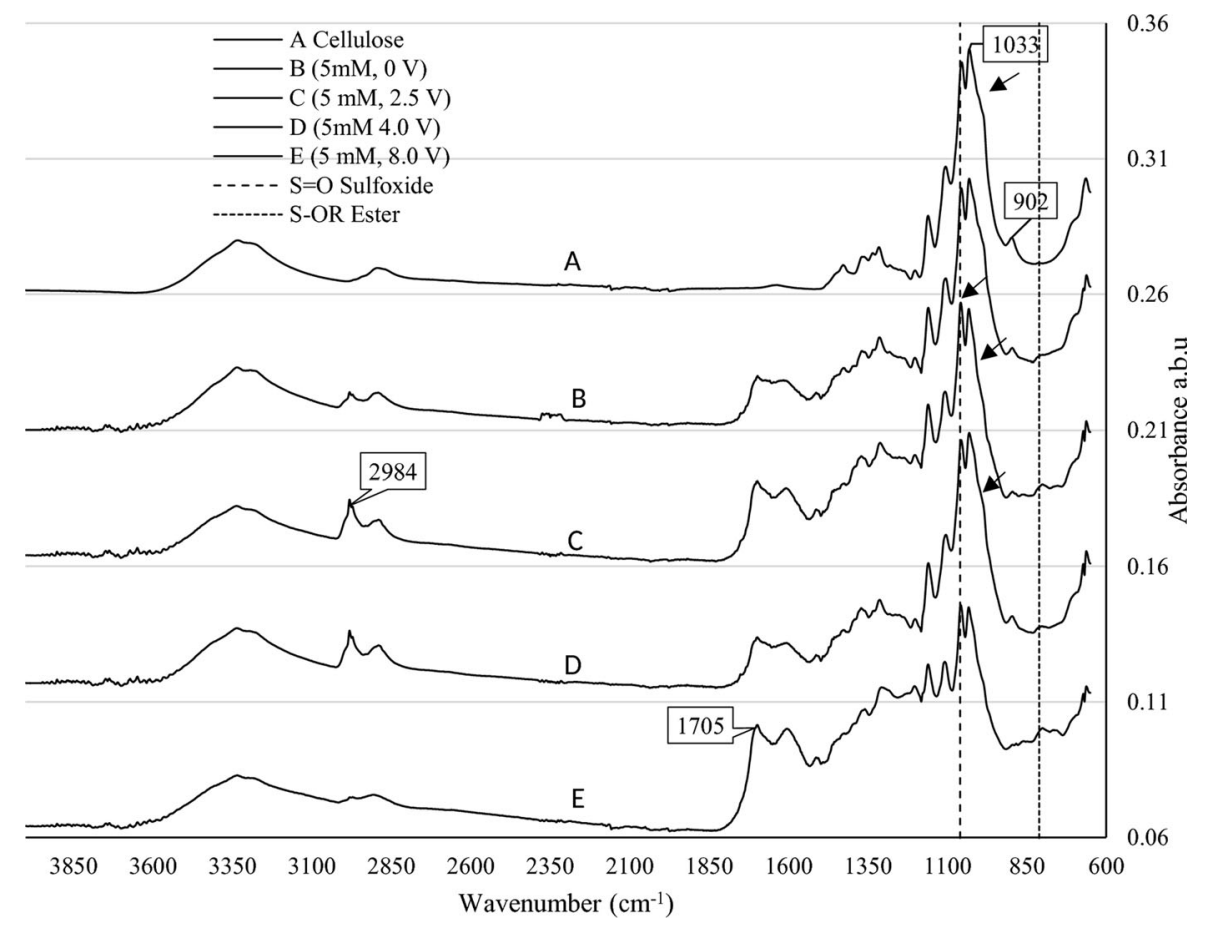

peak height at $1060 \mathrm{~cm}^{-1}$ became higher than $1030 \mathrm{~cm}^{-1}$ at $2.5 \mathrm{~V}$ potential, indicating that sulfoxide bond formation took place. The change in spectrums at 1030 and $1060 \mathrm{~cm}^{-1}$ (shoulder) with applied voltage was not observed in acid free reaction medium (Fig. 10). Change in the selectivity of product distributions by application of $2.5 \mathrm{~V}$ potential can be explained by the formation of sulfonate groups in solid residuals. Moreover, application of voltage resulted in the formation of carboxylic acid $(\mathrm{C}=\mathrm{O})$ functional group at stretching vibration of $1705 \mathrm{~cm}^{-1}$ (Nakhate and Yadav 2016). Primary alcohol group of cellulose could be oxidized via cyclic or acyclic ways that resulted in formation of carboxylic acid (Fig. 8), aldehyde and ketone functionality (Rajalaxmi et al.
2010). FTIR spectrum of 2.5 and $8.0 \mathrm{~V}$ (Fig. 9b, e) indicated that higher carbonyl band intensity was higher under applied voltage. This intensity was a sign of oxidation of primary or secondary alcohol group of cellulose to carboxylic acid functionality. FTIR spectrums of solid residual also supported the idea of catalytic effect of solid particle within the reaction medium under applied voltage because of formed sulfonated and carboxylic acid groups.

\section{Conclusion}

Hydrothermal electrolysis of MCC were carried out under constant potential range of $2.5 \mathrm{~V}-8.0 \mathrm{~V}$. 
Fig. 10 FTIR spectrum of solid residues of applied potential of $2.5,4.0$ and $8.0 \mathrm{~V}$ in acid free medium

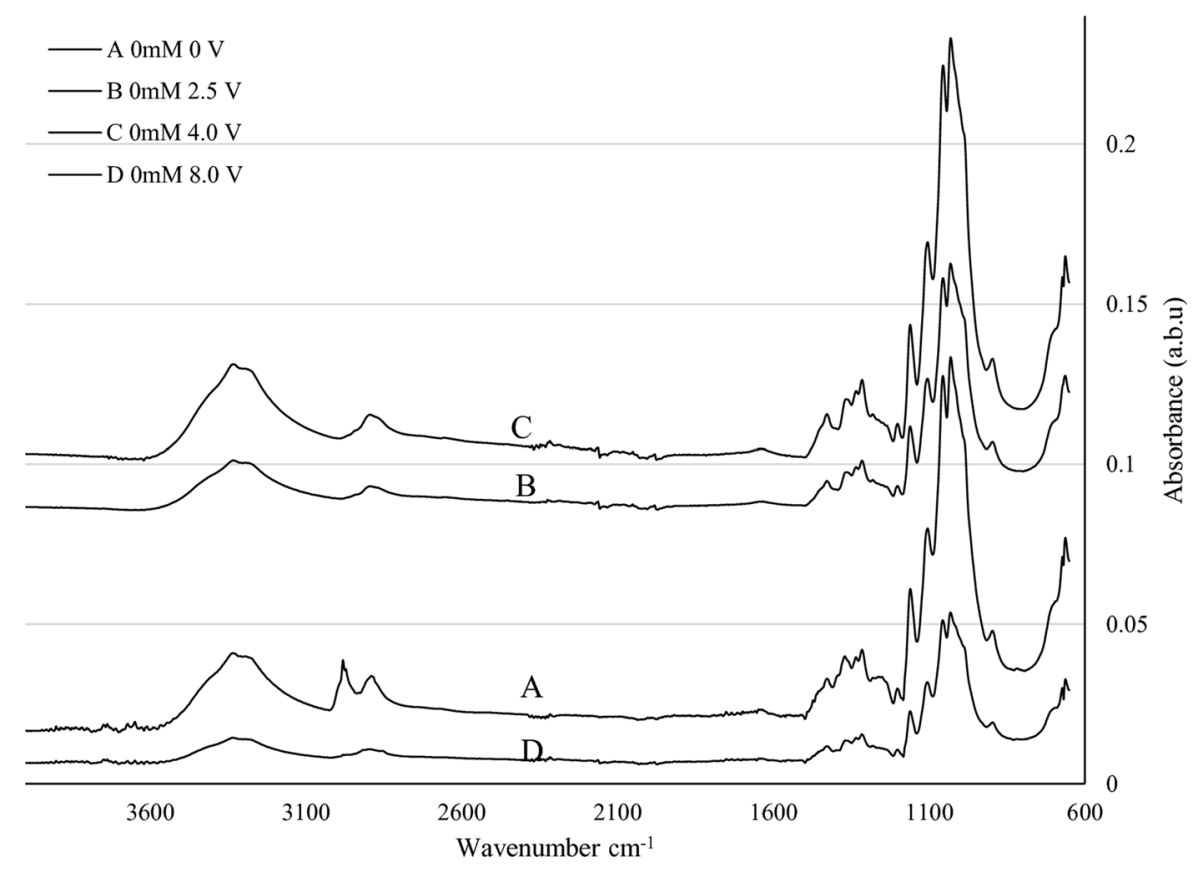

Formation of organic compounds (TOC) and product selectivity values were investigated in the presence and absence of electrolyte solution. As an electrolyte sulfuric acid was used in concentration of 5 and $25 \mathrm{mM}$. In the absence of electrolyte, application of voltage $(2.5$ and $4.0 \mathrm{~V})$ decreased TOC yield of reaction under hydrothermal conditions. Diverse effect was conducted with the redox reaction of ionic products water, which might hinder the protonation of $\beta$-glyosidic bond of cellulose under applied potential. In contrast, higher potential $8.0 \mathrm{~V}$ resulted in higher yield of TOC (13\%) showing that applied over potential might result in the formation of more reactive species as hydroxyl radical $\left(\mathrm{OH}^{\bullet-}\right)$ that could trigger the cleavage of $\beta$-glyosidic bond. Moreover, application of $8.0 \mathrm{~V}$ altered the decomposition mechanism to furfural with the increased selectivity of $13 \%$ in comparison to current free experiment (\%7). Application of $2.5 \mathrm{~V}$ in $5 \mathrm{mM}$ sulfuric acid solution also altered the TOC yield (54\%) but most dramatic changes were observed in selectivity values of 5-HMF (30\%) and levulinic acid (21\%). The change in the selectivity values of degradation products and the structural changes in solid residues of electrochemically reacted MCC was conducted by Fourier Transform Infrared Spectroscopy (FTIR) and it was found that MCC particles functionalized by carboxylic acid and sulfonated groups by the application of constant voltage to reaction medium. Under certain potential difference $(2.5 \mathrm{~V})$, functionalization of primary and secondary alcohol groups of cellulose to carboxylic acid and sulfoxide became more obvious in FTIR results. Thus, it was suggested that, functionalization of particles may alter the selectivity of decomposition products of cellulose since formation of sulfoxide and carboxylic acid functionality has catalytic effect on decomposition of cellulose.

Acknowledgments This research is financially supported by Marie Curie Career Integration Grants (FP7-PEOPLE-2012CIG) with a Project Number of PCIG11-GA-2012-321741. We would like to thank to two research centers at Izmir Institute of Technology for their support in product analysis: "Biotechnology and Bioengineering Research and Application Center" and "Environmental Reference Research and Development Center'.

\section{References}

Aida TM, Sato Y, Watanabe M, Tajima K, Nonaka T, Hattori H, Arai K (2007) Dehydration Of $D$-glucose in high temperature water at pressures up to $80 \mathrm{MPa}$. J Supercrit Fluid 40:381-388

Akin O, Yuksel A (2016) Novel hybrid process for the conversion of microcrystalline cellulose to value-added chemicals: part 1: process optimization. Cellulose 23:3475-3493. doi:10.1007/s10570-016-1054-3 
Asghari FS, Yoshida H (2008) Electrodecomposition in subcritical water using o-xylene as a model for benzene, toluene, ethylbenzene, and xylene pollutants. J Phys Chem A 112:7402-7410

Davis J, Baygents JC, Farrell J (2014) Understanding persulfate production at boron doped diamond film anodes. Electrochim Acta 150:68-74

dos Santos TR, Nilges P, Sauter W, Harnisch F, Schröder U (2015) Electrochemistry for the generation of renewable chemicals: electrochemical conversion of levulinic acid. Rsc Adv 5:26634

Jitaru M, Lowy DA, Toma M, Toma BC, Oniciu L (1997) Electrochemical reduction of carbon dioxide on flat metallic cathodes. J Appl Electrochem 27:875-889. doi:10. 1023/a:1018441316386

Kabyemela BM, Takigawa M, Adschiri T, Malaluan RM, Arai K (1998) Mechanism and kinetics of cellobiose decomposition in sub- and supercritical water. Ind Eng Chem Res 37:357-361. doi:10.1021/ie9704408

Kang SM, Ye J, Zhang Y, Chang J (2013) Preparation of biomass hydrochar derived sulfonated catalysts and their catalytic effects for 5-hydroxymethylfurfural production. Rsc Adv 3:7360-7366

Kilic E, Yilmaz S (2015) Fructose Dehydration to 5-Hydroxymethylfurfural over Sulfated TiO2-SiO2, Ti-SBA-15, $\mathrm{ZrO} 2, \mathrm{SiO} 2$, and Activated Carbon Catalysts. Ind Eng Chem Res 54:5220-5225

Li MH, Li WZ, Lu YJ, Jameel H, Chang HM, Ma LL (2017) High conversion of glucose to 5-hydroxymethylfurfural using hydrochloric acid as a catalyst and sodium chloride as a promoter in a water/gamma-valerolactone system. Rsc Adv 7:14330-14336

Luijkx GCA, van Rantwijk F, van Bekkum H (1993) Hydrothermal formation of 1,2,4-benzenetriol from 5-hydroxymethyl-2-furaldehyde and $D$-fructose. Carbohyd Res 242:131-139. doi:10.1016/0008-6215(93)80027-C

Marshall WL, Franck EU (1981) Ion product of water substance, O-Degrees-C-1000-Degrees-C, 1-10,000 bars-new international formulation and its background. J Phys Chem Ref Data 10:295-304

Nakhate AV, Yadav GD (2016) Synthesis and characterization of sulfonated carbon-based graphene oxide monolith by solvothermal carbonization for esterification and unsymmetrical ether formation. Acs Sustain Chem Eng 4: 1963-1973

Promdej C, Matsumura Y (2011) Temperature effect on hydrothermal decomposition of glucose in sub- and supercritical water. Ind Eng Chem Res 50:8492-8497. doi:10.1021/ie200298c
Qi XH, Guo HX, Li LY, Smith RL (2012) Acid-catalyzed dehydration of fructose into 5-hydroxymethylfurfural by cellulose-derived amorphous carbon. Chemsuschem 5:2215-2220

Rajalaxmi D, Jiang N, Leslie G, Ragauskas AJ (2010) Synthesis of novel water-soluble sulfonated cellulose. Carbohyd Res 345:284-290

Rossmeisl J, Logadottir A, Nørskov JK (2005) Electrolysis of water on (oxidized) metal surfaces. Chem Phys 319:178-184. doi:10.1016/j.chemphys.2005.05.038

Sasaki M, Fang Z, Fukushima Y, Adschiri T, Arai K (2000) Dissolution and hydrolysis of cellulose in subcritical and supercritical water. Ind Eng Chem Res 39:2883-2890

Sasaki M, Adschiri T, Arai K (2004) Kinetics of cellulose conversion at $25 \mathrm{MPa}$ in sub- and Supercritical water. AIChE J 50:192-202

Sasaki M, Yamamoto K, Goto M (2007) Reaction mechanism and pathway for the hydrothermal electrolysis of organic compounds. J Mater Cycles Waste 9:40-46

Sasaki M, Oshikawa T, Watanabe H, Wahyudiono, Goto M (2011) Reaction kinetics and mechanism for hydrothermal degradation and electrolysis of glucose for producing carboxylic acids. Res Chem Intermediat 37:457-466

Shen ZQ, Yu XM, Chen JZ (2016) Production of 5-Hydroxymethylfurfural from Fructose catalyzed by sulfonated bamboo-derived carbon prepared by simultaneous carbonization and sulfonation. BioResources 11:3094-3109

Swift TD, Bagia C, Choudhary V, Peklaris G, Nikolalds V, Vlachos DG (2014) Kinetics of homogeneous bronsted acid catalyzed fructose dehydration and 5-hydroxymethyl furfural rehydration: a combined experimental and computational study. Acs Catal 4:259-267

Xu B, Zhang BG, Li M, Huang WW, Chen N, Feng CP, Yao LJ (2014) Production of reducing sugars from corn stover by electrolysis. J Appl Electrochem 44:797-806

Yuksel A, Sasaki M, Goto M (2011) Electrolysis reaction pathway for lactic acid in subcritical water. Ind Eng Chem Res 50:728-734

Zhang YM, Peng Y, Yin XL, Liu ZH, Li G (2014) Degradation of lignin to BHT by electrochemical catalysis on $\mathrm{Pb} / \mathrm{PbO} 2$ anode in alkaline solution. J Chem Technol Biot 89:19541960

Zheng XJ, Gu XC, Ren Y, Zhi ZH, Lu XB (2016) Production of 5-hydroxymethyl furfural and levulinic acid from lignocellulose in aqueous solution and different solvents. Biofuel Bioprod Bior 10:917-931 